\title{
LARGE DEVIATIONS FOR RISK PROCESSES WITH REINSURANCE
}

\author{
CLAUDIO MACCI,* Università di Roma 'Tor Vergata' \\ GABRIELE STABILE, ${ }^{* *}$ Università di Roma 'La Sapienza'
}

\begin{abstract}
We consider risk processes with reinsurance. A general family of reinsurance contracts is allowed, including proportional and excess-of-loss policies. Claim occurrence is regulated by a classical compound Poisson process or by a Markov-modulated compound Poisson process. We provide some large deviation results concerning these two risk processes in the small-claim case. Finally, we derive the so-called Lundberg estimate for the ruin probabilities and present a numerical example.

Keywords: Large deviations; risk process; reinsurance; ruin probability; Lundberg estimate

2000 Mathematics Subject Classification: Primary 60F10; 91B30; 62P05
\end{abstract}

\section{Introduction}

\subsection{The model without reinsurance}

We consider the risk process $\left(X^{x}(t)\right)$ defined by

$$
X^{x}(t)=x+p t-S(t),
$$

where $x>0$ is the initial capital, $p>0$ is the (constant) premium rate, and the aggregate claims process $(S(t))$ is a compound Poisson process (in the classical case) or a Markovmodulated compound Poisson process (in the Markov-modulated case). More precisely, we have $S(t)=\sum_{k=1}^{N(t)} U_{k}$ and $N(t)=\sum_{k \geq 1} 1_{\left\{T_{k} \leq t\right\}}$, where $\left(U_{k}\right)$ is a sequence of positive random variables and $(N(t))$ is a counting process with points $\left(T_{k}\right)$; further details will be given when we present the classical case and the Markov-modulated case separately. Roughly speaking, in the Markov-modulated case, the claim intensity and claim size distribution depend on the evolution of a Markov chain with a finite state space; from the actuarial point of view, the Markov chain describes the environmental conditions that influence the phenomena, such as weather conditions in car insurance.

We assume that the random variables $\left(U_{k}\right)$ have finite expected values and that $S(t) / t$ converges to some limit value $\ell$ as $t \rightarrow \infty$. The (infinite-horizon) ruin probabilities $(\psi(x))_{x>0}$ are defined by

$$
\psi(x)=\mathrm{P}\left(\tau^{x}<\infty\right), \quad \text { where } \tau^{x}=\inf \left\{t \geq 0: X^{x}(t)<0\right\},
$$

and, in order to avoid the trivial case $\psi(x)=1$ for all $x>0$, the so-called net profit condition is required, i.e. $p=(1+\kappa) \ell$ for some relative safety loading $\kappa>0$.

Received 2 September 2005; revision received 6 April 2006.

* Postal address: Dipartimento di Matematica, Università di Roma 'Tor Vergata', Via della Ricerca Scientifica, I-00133 Rome, Italy. Email address: macci@mat.uniroma2.it

** Postal address: Dipartimento di Matematica per le Decisioni Economiche Finanziarie ed Assicurative, Università di Roma 'La Sapienza', Via del Castro Laurenziano 9, I-00161 Rome, Italy. Email address: gabriele.stabile@ uniroma1.it 


\subsection{Reinsurance policies}

In our model reinsurance is allowed, i.e. the insurance company may insure part of the risk at another company (the reinsurance company) in return for part of the premium, $p t$. A reinsurance policy is described by a measurable function $\mathcal{R}:[0, \infty) \times[0, \infty) \rightarrow[0, \infty)$, for which we use the notation $\mathcal{R}(t, \alpha) \equiv R_{t}(\alpha)$; for such a function we require the condition $0 \leq R_{t}(\alpha) \leq \alpha$ for all $t, \alpha \geq 0$. This means that $R_{t}(\alpha)$ is the part of the claim that the company pays when a claim of size $\alpha$ occurs at time $t$. Since the reinsurance policy is chosen dynamically, the premium rate for the reinsurer is in general not constant in time, as it is in the classical risk model. We denote by $q_{\mathcal{R}}(t)$ the premium paid up to time $t$ by the insurer to the reinsurer, and we shall see in detail below its determination. We assume that the reinsurer uses the expected value principle with relative safety loading $\eta>0$ for premium calculation. We shall point out below that it is interesting to consider $\eta>\kappa$, i.e. the case in which reinsurance is more expensive than insurance, since in any other case the insurer would reinsure the whole portfolio. In conclusion, the reserve process $\left(X_{\mathcal{R}}^{x}(t)\right)$ under the reinsurance policy $\mathcal{R}$ is defined by

$$
\begin{gathered}
X_{\mathcal{R}}^{x}(t)=x+p_{\mathcal{R}}(t)-S_{\mathcal{R}}(t), \quad \text { where } \\
S_{\mathcal{R}}(t)=\sum_{k=1}^{N(t)} R_{T_{k}}\left(U_{k}\right) \quad \text { and } \quad p_{\mathcal{R}}(t)=p t-q_{\mathcal{R}}(t) .
\end{gathered}
$$

\subsection{Large deviations and outline of the paper}

In this paper we present some large deviation results concerning the risk process under the reinsurance policy $\mathcal{R}$, with reference to the claim surplus process $\left(Z_{\mathcal{R}}(t)\right)$ defined by

$$
Z_{\mathcal{R}}(t)=x-X_{\mathcal{R}}^{x}(t)=S_{\mathcal{R}}(t)-p_{\mathcal{R}}(t) .
$$

In particular, we refer to the concept of a large deviation principle (LDP) (see, e.g. [4, pp. 4-5] for the definition). We present two kinds of LDP. The first one concerns the classical case (see Section 2) and is a sample-path large deviation result because it is an LDP on the space of càdlàg functions $D[0,1]$, i.e. those which are right continuous and have left limits. The second one concerns the Markov-modulated case (see Section 3) and is an LDP on $\mathbb{R}$. More precisely, in the first case we have

$$
\begin{aligned}
-\inf _{f \in B^{\circ}} I_{\mathcal{R}}(f) & \leq \liminf _{\alpha \rightarrow \infty} \frac{1}{\alpha} \log \mathrm{P}\left(\frac{Z_{\mathcal{R}}(\alpha \cdot)}{\alpha} \in B\right) \\
& \leq \limsup _{\alpha \rightarrow \infty} \frac{1}{\alpha} \log \mathrm{P}\left(\frac{Z_{\mathcal{R}}(\alpha \cdot)}{\alpha} \in B\right) \leq-\inf _{f \in \bar{B}} I_{\mathcal{R}}(f)
\end{aligned}
$$

for all Borel sets $B$ in $D[0,1]$, where $I_{\mathcal{R}}$ is the rate function, and in the second case we have

$$
\begin{aligned}
-\inf _{y \in B^{\circ}} \Lambda_{\mathcal{R}}^{*}(y) & \leq \liminf _{t \rightarrow \infty} \frac{1}{t} \log \mathrm{P}\left(\frac{Z_{\mathcal{R}}(t)}{t} \in B\right) \\
& \leq \limsup _{t \rightarrow \infty} \frac{1}{t} \log \mathrm{P}\left(\frac{Z_{\mathcal{R}}(t)}{t} \in B\right) \leq-\inf _{y \in \bar{B}} \Lambda_{\mathcal{R}}^{*}(y)
\end{aligned}
$$

for all Borel sets $B$ in $\mathbb{R}$, where $\Lambda_{\mathcal{R}}^{*}$ is the rate function. (We use the standard notation $B^{\circ}$ for the interior of $B$ and $\bar{B}$ for the closure of $B$.) It is useful to point out that $I_{\mathcal{R}}$ is a good rate 
function, i.e. the level sets of $I_{\mathcal{R}}$,

$$
\left\{f \in D[0,1]: I_{\mathcal{R}}(f) \leq c\right\} \quad(\text { for all } c>0),
$$

are compact sets.

The Markov-modulated case is a generalization of the classical case. The proof of the sample-path LDP in the classical case is based on Proposition 2.1, which is a known result. In the Markov-modulated case we do not have an analogous sample-path large deviation result, so we can only prove the LDP on $\mathbb{R}$.

In Section 4 we present some results for the ruin probabilities $\left(\psi_{\mathcal{R}}(x)\right)_{x>0}$ under the reinsurance policy $\mathcal{R}$. These are defined by

$$
\psi_{\mathcal{R}}(x)=\mathrm{P}\left(\tau_{\mathcal{R}}^{x}<\infty\right), \quad \text { where } \tau_{\mathcal{R}}^{x}=\inf \left\{t \geq 0: X_{\mathcal{R}}^{x}(t)<0\right\} .
$$

In Subsection 4.1 we prove the so-called Lundberg estimate for the ruin probabilities in (1.3); this estimate shows that, in a sense related to large deviations, $\psi_{\mathcal{R}}(x)$ decays exponentially as $x \rightarrow \infty$. Some comments and a numerical example are presented in Subsection 4.2.

\subsection{Hypothesis $(H)$ for the reinsurance policies}

We have in mind two different prototype examples of reinsurance policies (presented below), but in all the results in this paper we refer to the following condition.

Condition 1.1. (Hypothesis (H).) Let $R_{\infty}:[0, \infty) \rightarrow[0, \infty)$ be a measurable function. Then, for all $\varepsilon>0$, there exists a $t_{\varepsilon}$ such that for all $t \geq t_{\varepsilon}$ we have $\left|R_{t}(\alpha)-R_{\infty}(\alpha)\right| \leq \varepsilon \max \{\alpha, 1\}$ for all $\alpha \geq 0$.

In Condition 1.1 we could consider the bound $\alpha+1$, which is simpler (although larger) than $\max \{\alpha, 1\}$; in such a case some details presented in the paper have to be accordingly changed.

We remark that, when hypothesis $(\mathrm{H})$ holds, we have the pointwise convergence of $R_{t}$ to $R_{\infty}$ as $t \rightarrow \infty$ :

$$
\lim _{t \rightarrow \infty} R_{t}(\alpha)=R_{\infty}(\alpha) \quad \text { for all } \alpha \geq 0 .
$$

Indeed, for all $\varepsilon>0$ and all $\alpha \geq 0$, let $t_{\varepsilon, \alpha}$ be defined by $t_{\varepsilon, \alpha}:=t_{\varepsilon / \max \{\alpha, 1\}}$; then, for all $t \geq t_{\varepsilon, \alpha}$, we have

$$
\left|R_{t}(\alpha)-R_{\infty}(\alpha)\right| \leq \frac{\varepsilon}{\max \{\alpha, 1\}} \max \{\alpha, 1\}=\varepsilon .
$$

1.4.1. Prototype example 1: proportional policies. Let $R_{t}(\alpha)=b_{t} \alpha$ for some $b_{t} \in[0,1]$ and assume that $\lim _{t \rightarrow \infty} b_{t}=b_{\infty} \in[0,1]$. We check hypothesis $(\mathrm{H})$ as follows. Let $R_{\infty}(\alpha)=$ $b_{\infty} \alpha$. For all $\varepsilon>0$, there exists a $t_{\varepsilon}$ such that $\left|b_{t}-b_{\infty}\right| \leq \varepsilon$ for all $t \geq t_{\varepsilon}$; thus, for all $t \geq t_{\varepsilon}$,

$$
\left|R_{t}(\alpha)-R_{\infty}(\alpha)\right|=\left|b_{t}-b_{\infty}\right| \alpha \leq \varepsilon \alpha \leq \varepsilon \max \{\alpha, 1\} \quad \text { for all } \alpha \geq 0 .
$$

1.4.2. Prototype example 2: excess-of-loss policies. Let $R_{t}(\alpha)=\min \left\{a_{t}, \alpha\right\}$ for some $a_{t} \in$ $[0, \infty)$ and assume that $\lim _{t \rightarrow \infty} a_{t}=a_{\infty} \in[0, \infty)$. We check hypothesis (H) as follows. Let $R_{\infty}(\alpha)=\min \left\{a_{\infty}, \alpha\right\}$. For all $\varepsilon>0$, there exists a $t_{\varepsilon}$ such that $\left|a_{t}-a_{\infty}\right| \leq \varepsilon$ for all $t \geq t_{\varepsilon}$; thus, for all $t \geq t_{\varepsilon}$,

$$
\begin{array}{r}
\left|R_{t}(\alpha)-R_{\infty}(\alpha)\right|=\left|\min \left\{a_{t}, \alpha\right\}-\min \left\{a_{\infty}, \alpha\right\}\right| \leq\left|a_{t}-a_{\infty}\right| \leq \varepsilon \leq \varepsilon \max \{\alpha, 1\} \\
\text { for all } \alpha \geq 0
\end{array}
$$




\section{Classical case}

In this section we consider the model (1.1) with $(S(t))$ a compound Poisson process. Thus, we have $S(t)=\sum_{k=1}^{N(t)} U_{k}$ and $N(t)=\sum_{k \geq 1} 1_{\left\{T_{k} \leq t\right\}}$, where $\left(U_{k}\right)$ and $(N(t))$ are independent, the $U_{k}$ are independent and identically distributed, and $(N(t))$ is a Poisson process with intensity $\lambda$, i.e. the random variables $T_{k}-T_{k-1}$ are independent and identically exponentially distributed with expected value $1 / \lambda$.

We assume the following superexponential condition to hold for the random variables $\left(U_{k}\right)$.

Condition 2.1. (Hypothesis (S1).) $\mathrm{E}\left[\mathrm{e}^{\theta U_{1}}\right]<\infty$ for all $\theta \in \mathbb{R}$.

As a consequence of hypothesis ( $\mathrm{S} 1)$, the (common) expected value, $\mu$, of the random variables $\left(U_{k}\right)$ is finite and $S(t) / t$ converges to $\ell=\lambda \mu$ as $t \rightarrow \infty$. The reserve process $\left(X_{\mathcal{R}}^{x}(t)\right)$ under the reinsurance policy $\mathcal{R}$ is defined by (1.2) with

$$
p_{\mathcal{R}}(t)=p t-q_{\mathcal{R}}(t)=(1+\kappa) \lambda \mu t-(1+\eta) \lambda \int_{0}^{t}(\underbrace{\mathrm{E}\left[U_{1}\right]}_{=\mu}-\mathrm{E}\left[R_{S}\left(U_{1}\right)\right]) \mathrm{d} s ;
$$

some easy computations yield for this

$$
p_{\mathcal{R}}(t)=(1+\eta) \lambda \int_{0}^{t} \mathrm{E}\left[R_{S}\left(U_{1}\right)\right] \mathrm{d} s-(\eta-\kappa) \lambda \mu t .
$$

In all our results we assume that hypothesis $(\mathrm{H})$ holds; thus, we have (1.4), whence we obtain

$$
\lim _{t \rightarrow \infty} \frac{1}{t} \int_{0}^{t} \mathrm{E}\left[R_{S}\left(U_{1}\right)\right] \mathrm{d} s=\mathrm{E}\left[R_{\infty}\left(U_{1}\right)\right]
$$

and, so,

$$
\lim _{t \rightarrow \infty} \frac{p_{\mathcal{R}}(t)}{t}=\bar{p}_{\mathcal{R}}, \quad \text { where } \bar{p}_{\mathcal{R}}=(1+\eta) \lambda \mathrm{E}\left[R_{\infty}\left(U_{1}\right)\right]-(\eta-\kappa) \lambda \mu .
$$

The net profit condition for the insurance company under the reinsurance policy $\mathcal{R}$ is $\bar{p}_{\mathcal{R}}>$ $\lambda \mathrm{E}\left[R_{\infty}\left(U_{1}\right)\right]$, i.e.

$$
(1+\eta) \lambda \mathrm{E}\left[R_{\infty}\left(U_{1}\right)\right]-(\eta-\kappa) \lambda \mu>\lambda \mathrm{E}\left[R_{\infty}\left(U_{1}\right)\right]
$$

thus, after some easy computations, we obtain

$$
\mathrm{E}\left[R_{\infty}\left(U_{1}\right)\right] \geq\left[1-\frac{\kappa}{\eta}\right] \mu .
$$

We point out that (2.2) always holds when $\kappa \geq \eta$. We also need to consider the process $\left(\bar{Z}_{\mathcal{R}}(t)\right)$ defined by

$$
\bar{Z}_{\mathcal{R}}(t)=\sum_{k=1}^{N(t)} R_{\infty}\left(U_{k}\right)-\bar{p}_{\mathcal{R}} t .
$$

We remark that the process $\left(\bar{Z}_{\mathcal{R}}(t)\right)$ and the net profit condition presented above are generalizations of the analogous items presented in [6, Section 2] for proportional reinsurance policies; for instance, the process $\left(X_{t}^{b}\right)$ in [6, Equation (3)] is the analogue of $\left(\bar{Z}_{\mathcal{R}}(t)\right)$. The net profit is the same if we have $\left(\bar{Z}_{\mathcal{R}}(t)\right)$ in place of $\left(Z_{\mathcal{R}}(t)\right)$. Finally, we consider 
the rate function $I_{\mathcal{R}}$ defined by

$$
I_{\mathcal{R}}(f)= \begin{cases}\int_{0}^{1} \Lambda_{\mathcal{R}}^{*}(\dot{f}(t)) \mathrm{d} t & \text { if } f \in \mathrm{AC}_{0}[0,1] \\ \infty & \text { otherwise }\end{cases}
$$

where $\operatorname{AC}_{0}[0,1]$ is the family of all absolutely continuous functions defined on $[0,1]$ and starting at the origin,

$$
\Lambda_{\mathcal{R}}^{*}(y)=\sup _{\theta \in \mathbb{R}}\left\{\theta y-\Lambda_{\mathcal{R}}(\theta)\right\}, \quad \Lambda_{\mathcal{R}}(\theta)=\lambda\left(\mathrm{E}\left[\mathrm{e}^{\theta R_{\infty}\left(U_{1}\right)}\right]-1\right)-\bar{p}_{\mathcal{R}} \theta,
$$

and a dot denotes a time derivative.

Our aim is to prove Proposition 2.2, below, i.e. that $\left(Z_{\mathcal{R}}(\alpha \cdot) / \alpha\right)$ satisfies the LDP with rate function $I_{\mathcal{R}}$ as in (2.3). In order to do so we shall show that $\left(Z_{\mathcal{R}}(\alpha \cdot) / \alpha\right)$ is exponentially equivalent to $\left(\bar{Z}_{\mathcal{R}}(\alpha \cdot) / \alpha\right)$ as $\alpha \rightarrow \infty$ (see Definition 4.2.10 of [4]); then Proposition 2.2 will be proved by considering Theorem 4.2.13 of [4] and the next result (of [2]; see also [3] and the references cited therein).

Proposition 2.1. ([2].) Assume that $\mathrm{E}\left[\mathrm{e}^{\theta R_{\infty}\left(U_{1}\right)}\right]<\infty$ for all $\theta \in \mathbb{R}$ and that hypothesis $(H)$ holds. Then $\left(\bar{Z}_{\mathcal{R}}(\alpha \cdot) / \alpha\right)$ satisfies the LDP with rate function $I_{\mathcal{R}}$ as in (2.3). Moreover, the rate function $I_{\mathcal{R}}$ is good.

Some preliminaries are needed to prove Proposition 2.2. We shall use $\lfloor x\rfloor$ to denote the integer part of a real number $x$. Let $\left(A_{n}\right)$ be the sequence defined by

$$
A_{n}=\sum_{k=1}^{n}\left|R_{T_{k}}\left(U_{k}\right)-R_{\infty}\left(U_{k}\right)\right|
$$

and let us consider the following lemma.

Lemma 2.1. Assume that hypotheses $(S 1)$ and $(H)$ hold. Then $\lim _{n \rightarrow \infty}(1 / n) \log \mathrm{E}\left[\mathrm{e}^{\theta A_{n}}\right]=0$ for all $\theta>0$.

Proof. Fix $\theta>0$ arbitrarily. We have $\mathrm{E}\left[\mathrm{e}^{\theta A_{n}}\right] \geq 1$ for all $n \geq 1$ since $\theta>0$ and the random variable $A_{n}$ is nonnegative; thus, we can immediately say that $\lim _{n \rightarrow \infty}(1 / n) \log \mathrm{E}\left[\mathrm{e}^{\theta A_{n}}\right] \geq$ 0 , and will complete the proof by showing that $\lim _{\sup _{n \rightarrow \infty}}(1 / n) \log \mathrm{E}\left[\mathrm{e}^{\theta A_{n}}\right] \leq 0$.

The asymptotic estimate (2.6), below, is needed. Let $n \geq 1$ and $\rho, r, \varepsilon>0$ be arbitrarily fixed. We then have

$$
\mathrm{P}(N(r) \geq\lfloor n \varepsilon\rfloor) \leq \mathrm{e}^{-\rho\lfloor n \varepsilon\rfloor} \mathrm{E}\left[\mathrm{e}^{\rho N(r)}\right]=\exp \left(-\rho\lfloor n \varepsilon\rfloor+\lambda r\left(\mathrm{e}^{\rho}-1\right)\right)
$$

and, therefore, $\left.\lim \sup _{n \rightarrow \infty}(1 / n) \log \mathrm{P}(N(r)) \geq\lfloor n \varepsilon\rfloor\right) \leq-\rho \varepsilon$; thus, since $\rho>0$ is arbitrary,

$$
\lim _{n \rightarrow \infty} \frac{1}{n} \log \mathrm{P}(N(r) \geq\lfloor n \varepsilon\rfloor)=-\infty
$$

In conclusion, since $\mathrm{P}\left(T_{\lfloor n \varepsilon\rfloor} \leq r\right)=\mathrm{P}(N(r) \geq\lfloor n \varepsilon\rfloor)$, we obtain

$$
\lim _{n \rightarrow \infty} \frac{1}{n} \log \mathrm{P}\left(T_{\lfloor n \varepsilon\rfloor} \leq r\right)=-\infty .
$$

Furthermore, note that

$$
A_{n}=\sum_{k=1}^{n}\left|R_{T_{k}}\left(U_{k}\right)-R_{\infty}\left(U_{k}\right)\right| \leq \sum_{k=1}^{n}\left[R_{T_{k}}\left(U_{k}\right)+R_{\infty}\left(U_{k}\right)\right] \leq 2 \sum_{k=1}^{n} U_{k}
$$


thus,

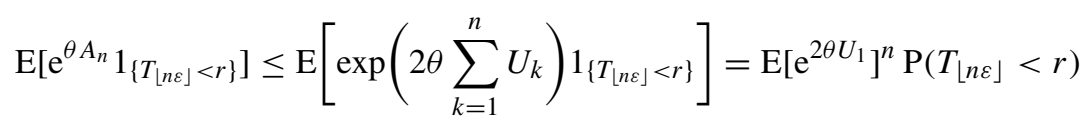

since $\theta>0,\left(U_{1}, \ldots, U_{n}, T_{\lfloor n \varepsilon\rfloor}\right)$ are independent, and $\left(U_{1}, \ldots, U_{n}\right)$ are independent and identically distributed, and we obtain

$$
\lim _{n \rightarrow \infty} \frac{1}{n} \log \mathrm{E}\left[\mathrm{e}^{\theta A_{n}} 1_{\left\{T_{\lfloor n \varepsilon\rfloor}<r\right\}}\right]=-\infty
$$

from (2.5) and hypothesis (S1).

Now let us consider the sum

$$
\mathrm{E}\left[\mathrm{e}^{\theta A_{n}}\right]=\mathrm{E}\left[\mathrm{e}^{\theta A_{n}} 1_{\left\{T_{\lfloor n \varepsilon\rfloor}<t_{\varepsilon}\right\}}\right]+\mathrm{E}\left[\mathrm{e}^{\theta A_{n}} 1_{\left\{T_{\lfloor n \varepsilon\rfloor} \geq t_{\varepsilon}\right\}}\right],
$$

where $t_{\varepsilon}$ is the value in hypothesis $(\mathrm{H})$. For the second term on the right-hand side, we obtain

$$
\begin{aligned}
\mathrm{E}\left[\mathrm{e}^{\theta A_{n}} 1_{\left\{T_{\lfloor n \varepsilon\rfloor} \geq t_{\varepsilon}\right\}}\right] & \leq \mathrm{E}\left[\exp \left(2 \theta \sum_{k=1}^{\lfloor n \varepsilon\rfloor-1} U_{k}\right) \exp \left(\theta \varepsilon \sum_{k=\lfloor n \varepsilon\rfloor}^{n} \max \left\{U_{k}, 1\right\}\right) 1_{\left\{T_{\lfloor n \varepsilon\rfloor} \geq t_{\varepsilon}\right\}}\right] \\
& \leq \mathrm{E}\left[\mathrm{e}^{2 \theta U_{1}}\right]^{\lfloor n \varepsilon\rfloor-1} \mathrm{E}\left[\mathrm{e}^{\theta \varepsilon \max \left\{U_{1}, 1\right\}}\right]^{n-\lfloor n \varepsilon\rfloor+1} .
\end{aligned}
$$

Thus, by (2.6) with $r=t_{\varepsilon}$, we have

$$
\limsup _{n \rightarrow \infty} \frac{1}{n} \log \mathrm{E}\left[\mathrm{e}^{\theta A_{n}}\right] \leq \varepsilon \log \mathrm{E}\left[\mathrm{e}^{2 \theta U_{1}}\right]+(1-\varepsilon) \log \mathrm{E}\left[\mathrm{e}^{\theta \varepsilon \max \left\{U_{1}, 1\right\}}\right] .
$$

In conclusion, we have $\lim \sup _{n \rightarrow \infty}(1 / n) \log \mathrm{E}\left[\mathrm{e}^{\theta A_{n}}\right] \leq 0$ since hypothesis (S1) holds and $\varepsilon>0$ can be chosen to be arbitrarily small.

Proposition 2.2. Assume that hypotheses $(S 1)$ and $(H)$ hold. Then $\left(Z_{\mathcal{R}}(\alpha \cdot) / \alpha\right)$ satisfies the $L D P$ with rate function $I_{\mathcal{R}}$ as in (2.3).

Proof. Proposition 2.1 provides the LDP of $\left(\bar{Z}_{\mathcal{R}}(\alpha \cdot) / \alpha\right)$ and the goodness of the rate function $I_{\mathcal{R}}$ in (2.3); indeed, when hypothesis (S1) holds we have $\mathrm{E}\left[\mathrm{e}^{\theta R_{\infty}\left(U_{1}\right)}\right]<\infty$ for all $\theta \in \mathbb{R}$. Thus, by Theorem 4.2 .13 of [4], we need only show that $\left(Z_{\mathcal{R}}(\alpha \cdot) / \alpha\right)$ and $\left(\bar{Z}_{\mathcal{R}}(\alpha \cdot) / \alpha\right)$ are exponentially equivalent as $\alpha \rightarrow \infty$, i.e. that

$$
\left.\lim _{\alpha \rightarrow \infty} \frac{1}{\alpha} \log \mathrm{P}\left(\frac{1}{\alpha} \sup _{t \in[0,1]}\left|Z_{\mathcal{R}}(\alpha t)-\bar{Z}_{\mathcal{R}}(\alpha t)\right|>\delta\right)=-\infty \quad \text { (for all } \delta>0\right) .
$$

Let $\delta>0$ be arbitrarily fixed. We have

$$
\begin{aligned}
& \left\{\frac{1}{\alpha} \sup _{t \in[0,1]}\left|Z_{\mathcal{R}}(\alpha t)-\bar{Z}_{\mathcal{R}}(\alpha t)\right|>\delta\right\} \\
& \quad \subset\left\{\frac{1}{\alpha} \sup _{t \in[0,1]}\left|p_{\mathcal{R}}(\alpha t)-\bar{p}_{\mathcal{R}} \alpha t\right|>\frac{\delta}{2}\right\} \cup\left\{\frac{1}{\alpha} \sup _{t \in[0,1]}\left|\sum_{k=1}^{N(\alpha t)} R_{T_{k}}\left(U_{k}\right)-R_{\infty}\left(U_{k}\right)\right|>\frac{\delta}{2}\right\} \\
& \quad \subset\left\{\frac{1}{\alpha} \sup _{t \in[0,1]}\left|p_{\mathcal{R}}(\alpha t)-\bar{p}_{\mathcal{R}} \alpha t\right|>\frac{\delta}{2}\right\} \cup\left\{\frac{1}{\alpha} \sup _{t \in[0,1]}^{N(\alpha t)} \sum_{k=1}^{N}\left|R_{T_{k}}\left(U_{k}\right)-R_{\infty}\left(U_{k}\right)\right|>\frac{\delta}{2}\right\} .
\end{aligned}
$$

Then

$$
\left\{\frac{1}{\alpha} \sup _{t \in[0,1]}\left|Z_{\mathcal{R}}(\alpha t)-\bar{Z}_{\mathcal{R}}(\alpha t)\right|>\delta\right\} \subset E_{1}^{\alpha} \cup E_{2}^{\alpha},
$$


where $E_{1}^{\alpha}=\left\{(1 / \alpha) \sup _{t \in[0,1]}\left|p_{\mathcal{R}}(\alpha t)-\bar{p}_{\mathcal{R}} \alpha t\right|>\delta / 2\right\}$ and $E_{2}^{\alpha}=\left\{A_{N(\alpha)} / \alpha>\delta / 2\right\}$, and, thus, from the union bound we obtain

$$
\mathrm{P}\left(\frac{1}{\alpha} \sup _{t \in[0,1]}\left|Z_{\mathcal{R}}(\alpha t)-\bar{Z}_{\mathcal{R}}(\alpha t)\right|>\delta\right) \leq \mathrm{P}\left(E_{1}^{\alpha}\right)+\mathrm{P}\left(E_{2}^{\alpha}\right) .
$$

In view of what follows it is useful to remark that in general $E_{1}^{\alpha}$ is a deterministic event; moreover, $E_{1}^{\alpha}=\varnothing$ and $(1 / \alpha) \sup _{t \in[0,1]}\left|p_{\mathcal{R}}(\alpha t)-\bar{p}_{\mathcal{R}} \alpha t\right| \leq \delta / 2$ are equivalent conditions. For sufficiently large $\alpha$ we have $E_{1}^{\alpha}=\varnothing$ because

$$
\lim _{\alpha \rightarrow \infty} \frac{1}{\alpha} \sup _{t \in[0,1]}\left|p_{\mathcal{R}}(\alpha t)-\bar{p}_{\mathcal{R}} \alpha t\right|=0
$$

indeed,

$$
\begin{aligned}
0 & \leq \frac{1}{\alpha} \sup _{t \in[0,1]}\left|p_{\mathcal{R}}(\alpha t)-\bar{p}_{\mathcal{R}} \alpha t\right| \\
& =\frac{(1+\eta) \lambda}{\alpha} \sup _{t \in[0,1]}\left|\int_{0}^{\alpha t} \mathrm{E}\left[R_{S}\left(U_{1}\right)\right] \mathrm{d} s-\mathrm{E}\left[R_{\infty}\left(U_{1}\right)\right] \alpha t\right| \\
& =\frac{(1+\eta) \lambda}{\alpha} \sup _{t \in[0,1]}\left|\int_{0}^{\alpha t}\left(\mathrm{E}\left[R_{S}\left(U_{1}\right)\right]-\mathrm{E}\left[R_{\infty}\left(U_{1}\right)\right]\right) \mathrm{d} s\right| \\
& \leq \frac{(1+\eta) \lambda}{\alpha} \sup _{t \in[0,1]} \int_{0}^{\alpha t}\left|\mathrm{E}\left[R_{S}\left(U_{1}\right)\right]-\mathrm{E}\left[R_{\infty}\left(U_{1}\right)\right]\right| \mathrm{d} s \\
& =\frac{(1+\eta) \lambda}{\alpha} \int_{0}^{\alpha}\left|\mathrm{E}\left[R_{S}\left(U_{1}\right)\right]-\mathrm{E}\left[R_{\infty}\left(U_{1}\right)\right]\right| \mathrm{d} s \\
& \leq \frac{(1+\eta) \lambda}{\alpha} \int_{0}^{\alpha} \mathrm{E}\left[\left|R_{S}\left(U_{1}\right)-R_{\infty}\left(U_{1}\right)\right|\right] \mathrm{d} s
\end{aligned}
$$

and, by (1.4), $\lim _{\alpha \rightarrow \infty}[(1+\eta) \lambda / \alpha] \int_{0}^{\alpha} \mathrm{E}\left[\left|R_{s}\left(U_{1}\right)-R_{\infty}\left(U_{1}\right)\right|\right] \mathrm{d} s=0$. Thus, for sufficiently large $\alpha,(2.8)$ becomes

$$
\mathrm{P}\left(\frac{1}{\alpha} \sup _{t \in[0,1]}\left|Z_{\mathcal{R}}(\alpha t)-\bar{Z}_{\mathcal{R}}(\alpha t)\right|>\delta\right) \leq \mathrm{P}\left(E_{2}^{\alpha}\right)
$$

and, since $E_{2}^{\alpha}=\left\{A_{N(\alpha)} / \alpha>\delta / 2\right\}$, the exponential equivalence (2.7) is proved if we can show that

$$
\limsup _{\alpha \rightarrow \infty} \frac{1}{\alpha} \log \mathrm{P}\left(A_{N(\alpha)}>\alpha \frac{\delta}{2}\right) \leq-\infty .
$$

To prepare to do so, let $\rho>0$ and a positive integer $K$ be arbitrarily fixed. Then, since $T_{n}$ is the sum of $n$ independent exponential random variables with mean $1 / \lambda$, we have

$$
\mathrm{P}\left(T_{K\lfloor\alpha\rfloor}<\alpha\right) \leq \mathrm{e}^{\rho \alpha} \mathrm{E}\left[\mathrm{e}^{-\rho T_{K\lfloor\alpha\rfloor}}\right]=\mathrm{e}^{\rho \alpha}\left(\frac{\lambda}{\lambda+\rho}\right)^{K\lfloor\alpha\rfloor}
$$

and, therefore,

$$
\lim _{\alpha \rightarrow \infty} \frac{1}{\alpha} \log \mathrm{P}\left(T_{K\lfloor\alpha\rfloor}<\alpha\right) \leq \rho+K \log \frac{\lambda}{\lambda+\rho} .
$$


Furthermore, we have $\left\{T_{K\lfloor\alpha\rfloor} \geq \alpha\right\}=\{N(\alpha) \leq K\lfloor\alpha\rfloor\}$; thus, since $\left(A_{n}\right)$ is nondecreasing, we obtain the inequality

$$
\mathrm{P}\left(A_{N(\alpha)}>\alpha \frac{\delta}{2}, T_{K\lfloor\alpha\rfloor} \geq \alpha\right) \leq \mathrm{P}\left(A_{K\lfloor\alpha\rfloor}>\alpha \frac{\delta}{2}\right) .
$$

Hence, for all $\theta>0$,

$$
\mathrm{P}\left(A_{N(\alpha)}>\alpha \frac{\delta}{2}, T_{K\lfloor\alpha\rfloor} \geq \alpha\right) \leq \mathrm{P}\left(A_{K\lfloor\alpha\rfloor}>\alpha \frac{\delta}{2}\right) \leq \mathrm{e}^{-\theta \alpha \delta / 2} \mathrm{E}\left[\mathrm{e}^{\theta A_{K}\lfloor\alpha\rfloor}\right]
$$

and, by Lemma $2.1, \lim \sup _{\alpha \rightarrow \infty}(1 / \alpha) \log \mathrm{P}\left(A_{N(\alpha)}>\alpha \delta / 2, T_{K\lfloor\alpha\rfloor} \geq \alpha\right) \leq-\theta \delta / 2$. In conclusion,

$$
\lim _{\alpha \rightarrow \infty} \frac{1}{\alpha} \log \mathrm{P}\left(A_{N(\alpha)}>\alpha \frac{\delta}{2}, T_{K\lfloor\alpha\rfloor} \geq \alpha\right)=-\infty
$$

holds since $\theta>0$ was arbitrarily chosen.

Now we are ready to prove (2.9). Let $\rho>0$ and a positive integer $K$ be arbitrarily fixed, as before. From the union bound we obtain

$$
\mathrm{P}\left(A_{N(\alpha)}>\alpha \frac{\delta}{2}\right) \leq \mathrm{P}\left(A_{N(\alpha)}>\alpha \frac{\delta}{2}, T_{K\lfloor\alpha\rfloor} \geq \alpha\right)+\mathrm{P}\left(T_{K\lfloor\alpha\rfloor}<\alpha\right) .
$$

Hence, by (2.10) and (2.11), we have

$$
\limsup _{\alpha \rightarrow \infty} \frac{1}{\alpha} \log \mathrm{P}\left(A_{N(\alpha)}>\alpha \frac{\delta}{2}\right) \leq \rho+K \log \frac{\lambda}{\lambda+\rho} .
$$

Moreover, for $K>\lambda$ we can set $\rho=K-\lambda$ and have

$$
\limsup _{\alpha \rightarrow \infty} \frac{1}{\alpha} \log \mathrm{P}\left(A_{N(\alpha)}>\alpha \frac{\delta}{2}\right) \leq K-\lambda-K \log \frac{K}{\lambda} .
$$

Equation (2.9) can be seen to hold by taking $K \rightarrow \infty$ on the right-hand side of this inequality.

\section{Markov-modulated case}

In this section we consider the Markov-modulated risk model of [10, Chapter 12, Section 3; see also Example 4, p. 506], i.e. the model (1.1) with $(S(t))$ a Markov-modulated compound Poisson process. Roughly speaking, let $J=(J(t))$ be an irreducible, continuous-time Markov chain with finite state space $E$ and assume that, in any finite time interval in which we have $J(t)=i$ for some $i \in E,(S(t))$ behaves like a compound Poisson process with claim intensity $\lambda_{i}$ and claim size distribution $G_{i}$. More precisely, we have $S(t)=\sum_{k=1}^{N(t)} U_{k}$ and $N(t)=\sum_{k \geq 1} 1_{\left\{T_{k} \leq t\right\}}$, where $\left(U_{k}\right)$ and $(N(t))$ are conditionally independent given $J,(N(t))$ is a Markov-modulated Poisson process, i.e. a doubly stochastic Poisson process with intensity $\left(\lambda_{J(t)}\right)$, the $U_{k}$ are independent given $J$, and, for all $k \geq 1$, the conditional distribution of $U_{k}$ given $J$ is $G_{J\left(T_{k}\right)}$.

In general we shall use the notation $\mathrm{E}_{i}[f(U)]$ to denote the expected value of a random variable $f(U)$, where $U$ is a random variable with distribution $G_{i}$.

In view of what follows it is useful to consider the following function $L: \mathbb{R}^{E} \rightarrow \mathbb{R}$; for details on this function, see $\left[1\right.$, Section 2]. Let $\boldsymbol{v}=\left[v_{i}\right]_{i \in E}$ be arbitrarily fixed and let $\left(p_{i j}\right)_{i, j \in E}$ be the intensity matrix of $J$; moreover, let us consider the matrix $\boldsymbol{P}(\boldsymbol{v})=\left(p_{i j}+\delta_{i j} v_{i}\right)_{i, j \in E}$, 
where

$$
p_{i j}+\delta_{i j} v_{i}= \begin{cases}p_{i j}+v_{i} & \text { if } i=j \\ p_{i j} & \text { if } i \neq j .\end{cases}
$$

Then the Perron-Frobenius theorem guarantees the existence of a simple and positive eigenvalue of the exponential matrix $\mathrm{e}^{\boldsymbol{P ( v )}}$, equal to the spectral radius of $\mathrm{e}^{\boldsymbol{P ( v )}} ; L(\boldsymbol{v})$ is defined to be the logarithm of this eigenvalue. It is also useful to recall the following known limit:

$$
L(\boldsymbol{v})=\lim _{t \rightarrow \infty} \frac{1}{t} \log \mathrm{E}\left[\exp \left(\int_{0}^{t} v_{J(s)} \mathrm{d} s\right)\right] \text { for all } \boldsymbol{v} \in \mathbb{R}^{E},
$$

whatever is the initial distribution of $J$. The function $L(\boldsymbol{v})$ is convex, nondecreasing with respect to each component of $\boldsymbol{v}$, and satisfies $\nabla L(\mathbf{0})=\pi$, where $\mathbf{0}$ is the null vector in $\mathbb{R}^{E}$ and $\pi=\left(\pi_{i}\right)_{i \in E}$ is the stationary distribution of $J$.

We assume the following condition, which is a generalization of hypothesis (S1) presented for the classical case, to hold.

Condition 3.1. (Hypothesis (S2).) For all $i \in E$, we have $\mathrm{E}_{i}\left[\mathrm{e}^{\theta U}\right]<\infty$ for all $\theta \in \mathbb{R}$.

As a consequence of hypothesis (S2), the expected values $\left(\mathrm{E}_{i}[U]\right)_{i \in E}$ are finite and $S(t) / t$ converges to $\ell=\sum_{i \in E} \pi_{i} \lambda_{i} \mathrm{E}_{i}[U]$ as $t \rightarrow \infty$. The reserve process $\left(X_{\mathcal{R}}^{x}(t)\right)$ under the reinsurance policy $\mathcal{R}$ is defined by (1.2) with

$p_{\mathcal{R}}(t)=p t-q_{\mathcal{R}}(t)=(1+\kappa) \sum_{i \in E} \pi_{i} \lambda_{i} \mathrm{E}_{i}[U] t-(1+\eta) \sum_{i \in E} \pi_{i} \lambda_{i} \int_{0}^{t}\left(\mathrm{E}_{i}[U]-\mathrm{E}_{i}\left[R_{S}(U)\right]\right) \mathrm{d} s$

some easy computations yield for this

$$
p_{\mathcal{R}}(t)=(1+\eta) \sum_{i \in E} \pi_{i} \lambda_{i} \int_{0}^{t} \mathrm{E}_{i}\left[R_{S}(U)\right] \mathrm{d} s-(\eta-\kappa) \sum_{i \in E} \pi_{i} \lambda_{i} \mathrm{E}_{i}[U] t .
$$

In all our results we assume that hypothesis $(\mathrm{H})$ holds. Thus, some of the results presented above can be adapted to the Markov-modulated case. Let us start with the limit (2.1) and the definition of $\bar{p}_{\mathcal{R}}$ : here

$$
\begin{gathered}
\lim _{t \rightarrow \infty} \frac{1}{t} \int_{0}^{t} \mathrm{E}_{i}\left[R_{S}(U)\right] \mathrm{d} s=\mathrm{E}_{i}\left[R_{\infty}(U)\right] \quad \text { for all } i \in E, \text { and } \\
\lim _{t \rightarrow \infty} \frac{p_{\mathcal{R}}(t)}{t}=\bar{p}_{\mathcal{R}}, \quad \text { where } \bar{p}_{\mathcal{R}}=(1+\eta) \sum_{i \in E} \pi_{i} \lambda_{i} \mathrm{E}_{i}\left[R_{\infty}(U)\right]-(\eta-\kappa) \sum_{i \in E} \pi_{i} \lambda_{i} \mathrm{E}_{i}[U] .
\end{gathered}
$$

The net profit condition for the insurance company under the reinsurance policy $\mathcal{R}$ is $\bar{p}_{\mathcal{R}}>$ $\sum_{i \in E} \pi_{i} \lambda_{i} \mathrm{E}_{i}\left[R_{\infty}(U)\right]$, i.e.

$$
(1+\eta) \sum_{i \in E} \pi_{i} \lambda_{i} \mathrm{E}_{i}\left[R_{\infty}(U)\right]-(\eta-\kappa) \sum_{i \in E} \pi_{i} \lambda_{i} \mathrm{E}_{i}[U]>\sum_{i \in E} \pi_{i} \lambda_{i} \mathrm{E}_{i}\left[R_{\infty}(U)\right] ;
$$


thus, after some easy computations, we obtain

$$
\sum_{i \in E} \pi_{i} \lambda_{i} \mathrm{E}_{i}\left[R_{\infty}(U)\right]>\left[1-\frac{\kappa}{\eta}\right] \sum_{i \in E} \pi_{i} \lambda_{i} \mathrm{E}_{i}[U] .
$$

We point out that (3.3) always holds when $\kappa \geq \eta$. Finally, let us consider the rate function $\Lambda_{\mathcal{R}}^{*}$ defined by

$$
\Lambda_{\mathcal{R}}^{*}(y)=\sup _{\theta \in \mathbb{R}}\left\{\theta y-\Lambda_{\mathcal{R}}(\theta)\right\}
$$

where

$$
\Lambda_{\mathcal{R}}(\theta)=L\left(\left[\lambda_{i}\left(\mathrm{E}_{i}\left[\mathrm{e}^{\theta R_{\infty}(U)}\right]-1\right)\right]_{i \in E}\right)-\bar{p}_{\mathcal{R}} \theta .
$$

Our aim is to prove Proposition 3.1, i.e. that $\left(Z_{\mathcal{R}}(t) / t\right)$ satisfies the LDP with rate function $\Lambda_{\mathcal{R}}^{*}$ as in (3.4). In order to do so we shall use the Gärtner-Ellis theorem (see [4, Chapter 2, Section 3]).

Proposition 3.1. Assume that hypotheses $(S 2)$ and $(H)$ hold. Then $\left(Z_{\mathcal{R}}(t) / t\right)$ satisfies the LDP with rate function $\Lambda_{\mathcal{R}}^{*}$ as in (3.4).

To prove Proposition 3.1, we need the following lemma.

Lemma 3.1. We have

$$
\mathrm{E}\left[\exp \left(\theta \sum_{k=1}^{N(t)} R_{T_{k}}\left(U_{k}\right)\right)\right]=\mathrm{E}\left[\exp \left(\int_{0}^{t} \lambda_{J_{s}}\left(\mathrm{E}_{J_{s}}\left[\mathrm{e}^{\theta R_{s}(U)}\right]-1\right) \mathrm{d} s\right)\right] .
$$

Proof. This formula can be proved following the lines of the proof of [8, Lemma 2.3] with $\varphi(s, u)=R_{S}(u) 1_{[0, t]}(s)$; more precisely, we have to consider the extension considered in [9, Lemma A.1].

Proof of Proposition 3.1. We want to apply the Gärtner-Ellis theorem and, for all $\theta \in \mathbb{R}$, we need to check the limit

$$
\lim _{t \rightarrow \infty} \frac{1}{t} \log \mathrm{E}\left[\mathrm{e}^{t \theta Z_{\mathcal{R}}(t) / t}\right]=\Lambda_{\mathcal{R}}(\theta) .
$$

First, note that by hypothesis (S2) we have $\mathrm{E}_{i}\left[\mathrm{e}^{\theta R_{\infty}(U)}\right]<\infty$ for all $i \in E$ and all $\theta \in \mathbb{R}$. Furthermore, we have

$$
\mathrm{E}\left[\mathrm{e}^{t \theta Z_{\mathcal{R}}(t) / t}\right]=\mathrm{E}\left[\exp \left(\theta \sum_{k=1}^{N(t)} R_{T_{k}}\left(U_{k}\right)\right)\right] \mathrm{e}^{-p_{\mathcal{R}}(t) \theta}
$$

whence we obtain

$$
\frac{1}{t} \log \mathrm{E}\left[\mathrm{e}^{t \theta Z_{\mathcal{R}}(t) / t}\right]=\frac{1}{t} \log \mathrm{E}\left[\exp \left(\theta \sum_{k=1}^{N(t)} R_{T_{k}}\left(U_{k}\right)\right)\right]-\frac{p_{\mathcal{R}}(t)}{t} \theta ;
$$


thus, by (3.2) and (3.5), we only have to check the following limit for all $\theta \in \mathbb{R}$ :

$$
\lim _{t \rightarrow \infty} \frac{1}{t} \log \mathrm{E}\left[\exp \left(\theta \sum_{k=1}^{N(t)} R_{T_{k}}\left(U_{k}\right)\right)\right]=L\left(\left[\lambda_{i}\left(\mathrm{E}_{i}\left[\mathrm{e}^{\theta R_{\infty}(U)}\right]-1\right)\right]_{i \in E}\right) .
$$

We can immediately say that (3.6) holds when $\theta=0$, since $L\left(\left[\lambda_{i}\left(\mathrm{E}_{i}\left[\mathrm{e}^{0 \times R_{\infty}(U)}\right]-1\right)\right]_{i \in E}\right)=$ $L(\mathbf{0})=0$.

In order to prove (3.6) when $\theta \neq 0$, we distinguish the cases $\theta>0$ and $\theta<0$. In both the cases we start from Lemma 2.1 with $t \geq t_{\varepsilon}$, where $t_{\varepsilon}$ is the value in hypothesis $(\mathrm{H})$, writing the result as

$$
\begin{aligned}
& \mathrm{E}\left[\exp \left(\theta \sum_{k=1}^{N(t)} R_{T_{k}}\left(U_{k}\right)\right)\right] \\
& \quad=\mathrm{E}\left[\exp \left(\int_{0}^{t_{\varepsilon}} \lambda_{J_{s}}\left(\mathrm{E}_{J_{S}}\left[\mathrm{e}^{\theta R_{s}(U)}\right]-1\right) \mathrm{d} s+\int_{t_{\varepsilon}}^{t} \lambda_{J_{S}}\left(\mathrm{E}_{J_{S}}\left[\mathrm{e}^{\theta R_{S}(U)}\right]-1\right) \mathrm{d} s\right)\right] .
\end{aligned}
$$

Case 1: $\theta>0$. Let $M(\theta)=\max _{i \in E} \lambda_{i}\left(E_{i}\left[\mathrm{e}^{\theta U}\right]-1\right)$. By (3.7), we then have

$$
\begin{aligned}
& \mathrm{E}\left[\exp \left(\int_{t_{\varepsilon}}^{t} \lambda_{J_{s}}\left(\mathrm{E}_{J_{s}}\left[\mathrm{e}^{\theta R_{s}(U)}\right]-1\right) \mathrm{d} s\right)\right] \\
& \quad \leq \mathrm{E}\left[\exp \left(\theta \sum_{k=1}^{N(t)} R_{T_{k}}\left(U_{k}\right)\right)\right] \\
& \quad \leq \mathrm{e}^{M(\theta) t_{\varepsilon}} \mathrm{E}\left[\exp \left(\int_{t_{\varepsilon}}^{t} \lambda_{J_{s}}\left(\mathrm{E}_{J_{s}}\left[\mathrm{e}^{\theta R_{S}(U)}\right]-1\right) \mathrm{d} s\right)\right] .
\end{aligned}
$$

Moreover, by hypothesis $(\mathrm{H})$, we obtain

$$
\begin{aligned}
& \mathrm{E}\left[\exp \left(\int_{t_{\varepsilon}}^{t} \lambda_{J_{s}}\left(\mathrm{E}_{J_{s}}\left[\exp \left(\theta\left(R_{\infty}(U)-\varepsilon \max \{U, 1\}\right)\right)\right]-1\right) \mathrm{d} s\right)\right] \\
& \quad \leq \mathrm{E}\left[\exp \left(\theta \sum_{k=1}^{N(t)} R_{T_{k}}\left(U_{k}\right)\right)\right] \\
& \quad \leq \mathrm{e}^{M(\theta) t_{\varepsilon}} \mathrm{E}\left[\exp \left(\int_{t_{\varepsilon}}^{t} \lambda_{J_{s}}\left(\mathrm{E}_{J_{s}}\left[\exp \left(\theta\left(R_{\infty}(U)+\varepsilon \max \{U, 1\}\right)\right)\right]-1\right) \mathrm{d} s\right)\right] .
\end{aligned}
$$

Then, by (3.1),

$$
\begin{aligned}
& L\left(\left[\lambda_{i}\left(\mathrm{E}_{i}\left[\exp \left(\theta\left(R_{\infty}(U)-\varepsilon \max \{U, 1\}\right)\right)\right]-1\right)\right]_{i \in E}\right) \\
& \quad \leq \liminf _{t \rightarrow \infty} \frac{1}{t} \log \mathrm{E}\left[\exp \left(\theta \sum_{k=1}^{N(t)} R_{T_{k}}\left(U_{k}\right)\right)\right] \\
& \quad \leq \limsup _{t \rightarrow \infty} \frac{1}{t} \log \mathrm{E}\left[\exp \left(\theta \sum_{k=1}^{N(t)} R_{T_{k}}\left(U_{k}\right)\right)\right] \\
& \quad \leq L\left(\left[\lambda_{i}\left(\mathrm{E}_{i}\left[\exp \left(\theta\left(R_{\infty}(U)+\varepsilon \max \{U, 1\}\right)\right)\right]-1\right)\right]_{i \in E}\right) .
\end{aligned}
$$

In conclusion, by the continuity of $L(\cdot),(3.6)$ holds since $\varepsilon>0$ is arbitrary. 
Case 2: $\theta<0$. For $\theta<0$ we simply adapt the procedure presented for the case in which $\theta>0$. Let $m=\min _{i \in E}\left(-\lambda_{i}\right)$. Then, by (3.7) and hypothesis $(\mathrm{H})$, we have

$$
\begin{aligned}
\mathrm{e}^{m t_{\varepsilon}} \mathrm{E}\left[\exp \left(\int_{t_{\varepsilon}}^{t} \lambda_{J_{s}}\left(\mathrm{E}_{J_{s}}\left[\exp \left(\theta\left(R_{\infty}(U)+\varepsilon \max \{U, 1\}\right)\right)\right]-1\right) \mathrm{d} s\right)\right] \\
\leq \mathrm{e}^{m t_{\varepsilon}} \mathrm{E}\left[\exp \left(\int_{t_{\varepsilon}}^{t} \lambda_{J_{s}}\left(\mathrm{E}_{J_{s}}\left[\mathrm{e}^{\theta R_{s}(U)}\right]-1\right) \mathrm{d} s\right)\right] \\
\leq \mathrm{E}\left[\exp \left(\theta \sum_{k=1}^{N(t)} R_{T_{k}}\left(U_{k}\right)\right)\right] \\
\leq \mathrm{E}\left[\exp \left(\int_{t_{\varepsilon}}^{t} \lambda_{J_{s}}\left(\mathrm{E}_{J_{s}}\left[\mathrm{e}^{\theta R_{s}(U)}\right]-1\right) \mathrm{d} s\right)\right] \\
\leq \mathrm{E}\left[\exp \left(\int_{t_{\varepsilon}}^{t} \lambda_{J_{s}}\left(\mathrm{E}_{J_{s}}\left[\exp \left(\theta\left(R_{\infty}(U)-\varepsilon \max \{U, 1\}\right)\right)\right]-1\right) \mathrm{d} s\right)\right] .
\end{aligned}
$$

Equation (3.6) can now be easily checked, using (3.1) in a suitable way and using the facts that $L(\cdot)$ is continuous and $\varepsilon>0$ is arbitrary.

Remark 3.1. The Markov-modulated case is a generalization of the classical case. This can be trivially explained by considering the set $E$ reduced to a single point. A more interesting way is to consider the following condition and some of its consequences: the distributions $\left(G_{i}\right)_{i \in E}$ are all the same, equalling $G$, say, and the values $\left(\lambda_{i}\right)_{i \in E}$ are all the same, equalling $\lambda$, say.

A first consequence is that $(S(t))$ is a compound Poisson process $S(t)=\sum_{k=1}^{N(t)} U_{k}$ of the type in the classical case, $G$ is the common distribution of the random variables $\left(U_{k}\right)$, and $(S(t))$ and $(J(t))$ are independent. Furthermore, hypothesis (S2) coincides with hypothesis (S1). Finally, $p_{\mathcal{R}}(t), \bar{p}_{\mathcal{R}}$, and $\Lambda_{\mathcal{R}}$ coincide with the items denoted by the same symbols in the classical case; for $\Lambda_{\mathcal{R}}$ this can be explained by noting that if, for some $v \in \mathbb{R}$, we have $v_{i}=v$ for all $i \in E$, then $L\left(\left[v_{i}\right]_{i \in E}\right)=v$.

\section{Results on ruin probabilities}

In this section we focus on the asymptotics of the ruin probability when the initial capital is large. In Subsection 4.1 we derive the so-called Lundberg estimate for the ruin probabilities in (1.3), i.e. the ruin probabilities under the reinsurance policy $\mathcal{R}$. In Subsection 4.2 we present some comments and a numerical example.

\subsection{Lundberg estimate}

The Lundberg estimate for the ruin probabilities $\left(\psi_{\mathcal{R}}(x)\right)_{x>0}$ will be proved in the next proposition. The Lundberg estimate is expressed as the limit (4.1); roughly speaking, this limit shows that $\psi_{\mathcal{R}}(x)$ decays exponentially as $x \rightarrow \infty$ in the fashion of large deviations.

Taking into account Remark 3.1, it is not restrictive to consider the Markov-modulated case; indeed, the classical case can then be seen as a particular case.

Proposition 4.1. Assume that hypotheses $(S 2)$ and $(H)$ hold. Also assume that

$$
\bar{p}_{\mathcal{R}}>\sum_{i \in E} \pi_{i} \lambda_{i} \mathrm{E}_{i}\left[R_{\infty}(U)\right]>0 .
$$


Then there exists a $w_{\mathcal{R}}>0$ such that $\Lambda_{\mathcal{R}}\left(w_{\mathcal{R}}\right)=0$ and

$$
\lim _{x \rightarrow \infty} \frac{1}{x} \log \psi_{\mathcal{R}}(x)=-w_{\mathcal{R}}
$$

Proof. We have $\Lambda_{\mathcal{R}}^{\prime}(0)<0$ by (3.5), $\nabla L(\mathbf{0})=\pi$, and the net profit condition $\bar{p}_{\mathcal{R}}>$ $\sum_{i \in E} \pi_{i} \lambda_{i} \mathrm{E}_{i}\left[R_{\infty}(U)\right]$. Moreover,

$$
\Lambda_{\mathcal{R}}(\theta) \geq \sum_{i \in E} \pi_{i} \lambda_{i}\left(\mathrm{E}_{i}\left[\mathrm{e}^{R_{\infty}(U) \theta}\right]-1\right)-\bar{p}_{\mathcal{R}} \theta
$$

by the convexity of $L$ and by $\nabla L(\mathbf{0})=\pi$, so the right-hand side of the displayed inequality diverges as $\theta \rightarrow \infty$ since $\pi_{i}, \lambda_{i}>0$ for all $i \in E$ and since at least one of the functions $\left(\mathrm{E}_{i}\left[\mathrm{e}^{R_{\infty}(U) \theta}\right]-1\right)_{i \in E}$ diverges by the hypothesis that $\sum_{i \in E} \pi_{i} \lambda_{i} \mathrm{E}_{i}\left[R_{\infty}(U)\right]>0$. Thus, $\Lambda_{\mathcal{R}}(\theta)$ diverges as $\theta \rightarrow \infty$. In conclusion, the existence of $w_{\mathcal{R}}$ is guaranteed by the convexity of $\Lambda_{\mathcal{R}}$ and the facts that $\Lambda_{\mathcal{R}}^{\prime}(0)<0, \lim _{\theta \rightarrow \infty} \Lambda_{\mathcal{R}}(\theta)=\infty$, and $\Lambda_{\mathcal{R}}(\theta)<\infty$ for all $\theta \in \mathbb{R}$ (the latter statement follows from hypothesis (S2)).

In order to prove (4.1) we refer to [5, Corollary 2.3 and Lemma 2.1] (taking linear scaling functions); thus, we have to check both that $\Lambda_{\mathcal{R}}^{*}$ is continuous at every point of $(0, \infty)$ and that the following inequality holds, where $Z_{\mathcal{R}}^{*}(n)=\sup _{0 \leq r<1} Z_{\mathcal{R}}(n+r)$ :

$$
\limsup _{n \rightarrow \infty} \frac{1}{n} \log \mathrm{E}\left[\mathrm{e}^{\theta\left(Z_{\mathcal{R}}^{*}(n)-Z_{\mathcal{R}}(n)\right)}\right] \leq 0 \quad \text { for all } \theta>0 .
$$

The function $\Lambda_{\mathcal{R}}^{*}$ is convex and finite on the set $\left\{\Lambda_{\mathcal{R}}^{\prime}(\theta): \theta \in \mathbb{R}\right\}=\left(-\bar{p}_{\mathcal{R}}, \infty\right)$; thus, $\Lambda_{\mathcal{R}}^{*}$ is continuous on this open set and, in particular, on $(0, \infty) \subset\left(-\bar{p}_{\mathcal{R}}, \infty\right)$. To prove (4.2), first note that

$$
Z_{\mathcal{R}}^{*}(n)-Z_{\mathcal{R}}(n)=\sup _{0 \leq r<1}\left\{S_{\mathcal{R}}(n+r)-S_{\mathcal{R}}(n)-\left(p_{\mathcal{R}}(n+r)-p_{\mathcal{R}}(n)\right)\right\} .
$$

Moreover,

$$
S_{\mathcal{R}}(n+r)-S_{\mathcal{R}}(n)=\sum_{k=N(n)+1}^{N(n+r)} R_{T_{k}}\left(U_{k}\right) \leq \sum_{k=N(n)+1}^{N(n+r)} U_{k}
$$

and

$$
\begin{aligned}
p_{\mathcal{R}}(n+r)-p_{\mathcal{R}}(n) & =\underbrace{(1+\eta) \sum_{i \in E} \pi_{i} \lambda_{i} \int_{n}^{n+r} \mathrm{E}_{i}\left[R_{S}(U)\right] \mathrm{d} s}_{\geq 0}-(\eta-\kappa) \sum_{i \in E} \pi_{i} \lambda_{i} \mathrm{E}_{i}[U] r \\
& \geq-(\eta-\kappa) \sum_{i \in E} \pi_{i} \lambda_{i} \mathrm{E}_{i}[U] r,
\end{aligned}
$$

whence we obtain (using the notation $z^{+}=\max \{z, 0\}$ )

$$
Z_{\mathcal{R}}(n+r)-Z_{\mathcal{R}}(n) \leq \sum_{k=N(n)+1}^{N(n+1)} U_{k}+(\eta-\kappa)^{+} \sum_{i \in E} \pi_{i} \lambda_{i} \mathrm{E}_{i}[U] \quad \text { for all } r, 0 \leq r<1 .
$$

Now let $\theta>0$ be arbitrarily fixed. We then have

$$
\mathrm{E}\left[\mathrm{e}^{\theta\left(Z_{\mathcal{R}}^{*}(n)-Z_{\mathcal{R}}(n)\right)}\right] \leq \mathrm{E}\left[\exp \left(\theta \sum_{k=N(n)+1}^{N(n+1)} U_{k}\right)\right] \exp \left(\theta(\eta-\kappa)^{+} \sum_{i \in E} \pi_{i} \lambda_{i} \mathrm{E}_{i}[U]\right) .
$$


Moreover, by Lemma 3.1 (slightly modified), we have

$$
\mathrm{E}\left[\exp \left(\theta \sum_{k=N(n)+1}^{N(n+1)} U_{k}\right)\right]=\mathrm{E}\left[\exp \left(\int_{n}^{n+1} \lambda_{J_{s}}\left(\mathrm{E}_{J_{s}}\left[\mathrm{e}^{\theta U}\right]-1\right) \mathrm{d} s\right)\right]
$$

and, if we let $M(\theta)=\max _{i \in E} \lambda_{i}\left(E_{i}\left[\mathrm{e}^{\theta U}\right]-1\right)$, as in the proof of Proposition 3.1, we obtain

$$
\mathrm{E}\left[\exp \left(\int_{n}^{n+1} \lambda_{J_{s}}\left(\mathrm{E}_{J_{s}}\left[\mathrm{e}^{\theta U}\right]-1\right) \mathrm{d} s\right)\right] \leq \mathrm{e}^{M(\theta)} .
$$

In conclusion,

$$
\mathrm{E}\left[\mathrm{e}^{\theta\left(Z_{\mathcal{R}}^{*}(n)-Z_{\mathcal{R}}(n)\right)}\right] \leq \exp \left(M(\theta)+\theta(\eta-\kappa)^{+} \sum_{i \in E} \pi_{i} \lambda_{i} \mathrm{E}_{i}[U]\right)
$$

and (4.2) holds since $M(\theta)<\infty$ by hypothesis (S2).

\subsection{Comments and a numerical example}

In this subsection we present some comments and a numerical example. In particular, taking into account the prototype examples presented at the end of Section 1, we mainly refer to proportional and excess-of-loss policies.

In the classical case, Schmidli [12] provided the Cramér-Lundberg approximation for proportional reinsurance strategies, which is an estimate sharper than the one presented in Proposition 4.1. Moreover, he asserted that other types of reinsurance can be treated similarly. For Markov-modulated risk processes, Hald and Schmidli [6, Section 4.2] treated the problem of how to calculate the proportional reinsurance strategy maximizing the adjustment coefficient. As far as the current authors are aware, there are no asymptotic results for Markov-modulated risk processes with excess-of-loss reinsurance.

A question of interest is in the choice of a dynamic reinsurance strategy to minimize the infinite-time ruin probability. In the case in which the risk process is approximated by a Brownian motion with drift, Schmidli [11] explicitly determined the optimal proportional reinsurance policy and the corresponding ruin probability function. The optimal retention level turns out to be a constant. In the case of the classical Cramér-Lundberg risk process, Schmidli [11] and Hipp and Vogt [7] analysed proportional reinsurance and excess-of-loss reinsurance, respectively. They proved the existence of a smooth solution to the HamiltonBellman-Jacobi equation as well as a verification theorem, but it seems that no explicit solution to the Hamilton-Bellman-Jacobi equation exists. In both papers it was conjectured that for exponentially distributed claim sizes the optimal reinsurance strategy becomes constant for large values of the initial capital. Thus, in general, it is hard to find an explicit solution to the control problem for the Cramér-Lundberg risk process.

The authors of a number of papers have focused their analyses on giving asymptotic results for the ruin probability. Waters [14] considered constant reinsurance strategies. He found that in the case of proportional reinsurance there exists a unique constant strategy that maximizes the adjustment coefficient. In the case of excess-of-loss reinsurance strategies, he argued that the same result holds if the premium is calculated according to the expected value principle. Schmidli studied the asymptotics for risk processes under optimal proportional reinsurance in both the small-claim case (see [12]) and the large-claim case (see [13]). In both cases, he provided the Cramér-Lundberg approximation as well as demonstrating the convergence of the 
optimal strategies. In particular, in the small-claim case he proved that the optimal reinsurance strategy converges to the asymptotically optimal strategy as the initial capital increases to infinity. In conclusion, in light of this discussion, the existence of the limit of the strategies $R_{t}$ as $t \rightarrow \infty$ (i.e. the strategy $R_{\infty}$ in hypothesis (H); see (1.4)) may be considered realistic.

It is interesting to determine the asymptotically optimal reinsurance strategy, that is, taking into account Proposition 4.1, the reinsurance strategy $\mathcal{R}$ that maximizes the adjustment coefficient $w_{\mathcal{R}}$. First consider the complete reinsurance case, where all the claims are entirely paid by the reinsurer (obviously hypothesis $(\mathrm{H})$ holds in this case; moreover, this can be seen both as a proportional policy and as an excess-of-loss policy):

$$
R_{t}^{\mathrm{c}}(\alpha)=0 \quad \text { for all } t, \alpha \geq 0 .
$$

The reserve process (1.2) becomes

$$
X_{\mathcal{R}^{\mathrm{c}}}^{x}(t)=x+(\kappa-\eta) \sum_{i \in E} \pi_{i} \lambda_{i} \mathrm{E}_{i}[U] t .
$$

Notice that if $\kappa \geq \eta$ then $\psi_{\mathcal{R}^{c}}(x)=0$ for all $x>0$. Thus, as pointed out in [6, Section 2] for proportional policies in the classical case, the inequality $\kappa \geq \eta$ leads to a trivial situation because the reinsurance policy (4.3) minimizes the ruin probability.

In conclusion, it is interesting instead to consider the inequality $\eta>\kappa$, i.e. the case in which reinsurance is more expensive than insurance. We have already pointed out that $\kappa \geq \eta$ trivially provides (2.2) in the classical case and (3.3) in the Markov-modulated case, when hypothesis $(\mathrm{H})$ holds.

It is in general hard to maximize the adjustment coefficient. Here we present a numerical example and consider proportional and excess-of-loss policies.

Example 4.1. Let $J$ be a two-state Markov chain and, thus, let $E=\{1,2\}$. Let $\lambda_{1}=1$ and $\lambda_{2}=2$ be the claim intensities and let $G_{1}$ and $G_{2}$ be the claim size distributions, which are both assumed to be exponential and to have respective expected values 1 and 2 . Let

$$
\left(\begin{array}{ll}
p_{11} & p_{12} \\
p_{21} & p_{22}
\end{array}\right)=\left(\begin{array}{l}
-1+1 \\
+1-1
\end{array}\right)
$$

be the intensity matrix of $J$. The corresponding stationary distribution is then $\left(\pi_{1}, \pi_{2}\right)=\left(\frac{1}{2}, \frac{1}{2}\right)$. Finally, let $\eta=5$ and $\kappa=4$ be the relative safety loading for the reinsurer and the insurer, respectively.

In Figure 1 we have depicted the adjustment coefficient $w_{\mathcal{R}}$ as a function of the retention level in the case of proportional reinsurance and in the case of excess-of-loss reinsurance. In both cases, the graphs suggest that $w_{\mathcal{R}}$ is a unimodal function of the retention level.

We point out that $w_{\mathcal{R}}=0$ when (3.3) fails to hold (or, in the classical case, when (2.2) fails to hold). In the proportional case, (3.3) is

$$
\frac{1}{2}\left[1 \times b_{\infty}+2 \times 2 b_{\infty}\right]>\left[1-\frac{4}{5}\right] \frac{1}{2}[1 \times 1+2 \times 2], \quad \text { i.e. } b_{\infty}>0.2 .
$$

In the excess-of-loss case, (3.3) is

$$
\frac{1}{2}\left[1 \times\left(1-\mathrm{e}^{-a_{\infty}}\right)+2 \times 2\left(1-\mathrm{e}^{-a_{\infty} / 2}\right)\right]>\left[1-\frac{4}{5}\right] \frac{1}{2}[1 \times 1+2 \times 2]
$$

and, after some easy computations, we obtain

$$
\mathrm{e}^{-a_{\infty} / 2}<2 \sqrt{2}-2, \quad \text { i.e. } a_{\infty}>-2 \log (2 \sqrt{2}-2)=0.38 .
$$



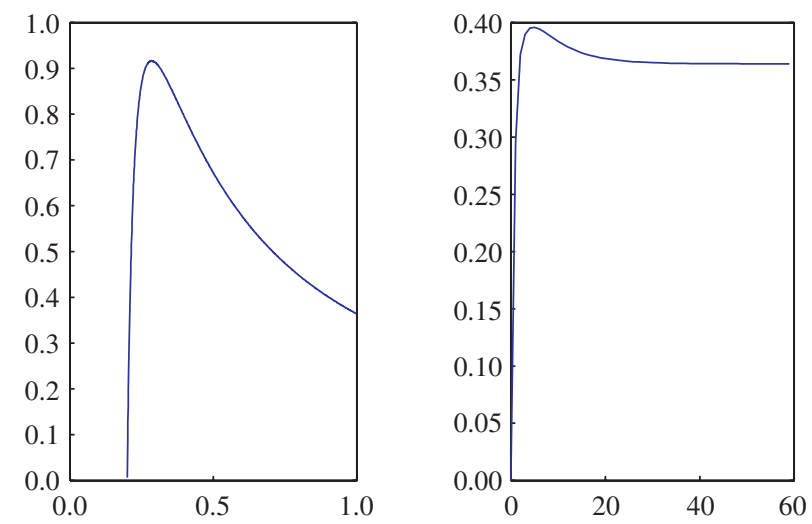

FIGURE 1: The adjustment coefficient as a function of the retention level $b_{\infty}$, in the case of proportional reinsurance (left), and $a_{\infty}$, in the case of excess-of-loss reinsurance (right).

\section{Acknowledgements}

This work has been partially supported by MURST project 'Metodi Stocastici in Finanza Matematica'. We thank the referee for some valuable comments and suggestions, which led to an improvement of both the content and the presentation of the paper.

\section{References}

[1] Baldi, P. And Piccioni, M. (1999). A representation formula for the large deviation rate function for the empirical law of a continuous time Markov chain. Statist. Prob. Lett. 41, 107-115.

[2] Borovkov, A. A. (1967). Boundary value problems for random walks and large deviations in function spaces. Theory Prob. Appl. 12, 575-595.

[3] De Acosta, A. (1994). Large deviations for vector-valued Lévy processes. Stoch. Process. Appl. 51, $75-115$.

[4] Dembo, A. and Zeitouni, O. (1993). Large Deviations Techniques and Applications. Jones and Bartlett, Boston, MA.

[5] Duffield, N. G. And O'Connell, N. (1995). Large deviations and overflow probabilities for a single server queue, with applications. Math. Proc. Camb. Phil. Soc. 118, 363-374.

[6] Hald, M. And Schmidli, H. (2004). On the maximisation of the adjustment coefficient under proportional reinsurance. ASTIN Bull. 34, 75-83.

[7] Hipp, C. And Vogt, M. (2003). Optimal dynamic XL reinsurance. ASTIN Bull. 33, 193-207.

[8] Macci, C. AND Torrisi, G. L. (2004). Asymptotic results for perturbed risk processes with delayed claims. Insurance Math. Econom. 34, 307-320.

[9] Macci, C., Stabile, G. AND ToRrisi, G. L. (2005). Lundberg parameters for non standard risk processes. Scand. Actuarial J. 2005, 417-432.

[10] Rolski, T., Schmidli, H., Schmidt, V. and Teugels, J. L. (1999). Stochastic Processes for Insurance and Finance. John Wiley, Chichester.

[11] Schmidi, H. (2001). Optimal proportional reinsurance policies in a dynamic setting. Scand. Actuarial J. 2001, $55-68$.

[12] Schmidli, H. (2002). Asymptotics of ruin probabilities for risk processes under optimal reinsurance policies: the small claim case. Working Paper 180, Laboratory of Actuarial Mathematics, University of Copenhagen.

[13] SchmiduI, H. (2004). Asymptotics of ruin probabilities for risk processes under optimal reinsurance and investment policies: the large claim case. Queueing Systems 46, 149-157.

[14] Waters, H. R. (1983). Some mathematical aspects of reinsurance. Insurance Math. Econom. 2, 17-26. 